\title{
Terahertz Detection Using Nanorectifiers
}

\author{
Shahrir Rizal Kasjoo and Ai Min Song, Senior Member, IEEE
}

\begin{abstract}
We report on the low-temperature detection of free-space radiation at $1.5 \mathrm{THz}$ using a unipolar nanodiode, known as the self-switching diode (SSD), coupled with a spiral microantenna. The SSD, based on an asymmetric nanochannel, has a diode-like characteristic that can be utilized in rectifying high-frequency electrical signals. The truly planar structure of the SSD not only provides intrinsically low parasitic capacitance that enables rectification at ultrahigh speed, but also allows the fabrication of a large SSD array in parallel without the need for interconnection layers. The extrinsic voltage responsivity of the SSD-based detector achieved was $\sim 15.6 \mathrm{~V} / \mathrm{W}$, but the estimated intrinsic voltage responsivity was $\sim 45 \mathrm{kV} / \mathrm{W}$.
\end{abstract}

Index Terms-Diode, nanotechnology, terahertz (THz) radiation.

\section{INTRODUCTION}

$\mathbf{T}$ ERAHERTZ $(\mathrm{THz})$ refers to the electromagnetic radiation in the frequency range from 0.1 to $10 \mathrm{THz}$. In the last two decades, interest in $\mathrm{THz}$ technology has grown steadily, given its outstanding potential in many applications, including telecommunication, defense and security, medical and healthcare, and material research [1]-[3]. In pathology, for example, $\mathrm{THz}$ radiation is able to distinguish between cancerous and healthy tissues based on their biological and chemical compositions [3].

In this letter, we present the $\mathrm{THz}$ operation at a low temperature of a device referred to as the self-switching diode (SSD), which is based on geometrical symmetry breaking of a semiconductor nanochannel [4]. Previous experiments have shown that the SSD worked at microwave and $\mathrm{THz}$ frequencies, in which the SSDs were coupled to a microwave waveguide or bow-tie antenna [5]-[7]. The bow-tie antenna is suitable to receive linearly polarized free-space $\mathrm{THz}$ radiations. For many applications, such as radio astronomy and $\mathrm{THz}$ imaging, the $\mathrm{THz}$ waves are not linearly polarized. Furthermore, although it is convenient to carry out and utilize $\mathrm{THz}$ waves at room temperature, $\mathrm{THz}$ detectors are often cooled to low temperatures to increase the signal-to-noise ratio. As such, we have in this letter fabricated SSDs with a spiral antenna [Fig. 1(a)]

Manuscript received August 15, 2013; revised September 22, 2013 and October 2, 2013; accepted October 5, 2013. Date of publication October 28, 2013; date of current version November 20, 2013. This work was supported in part by the EU FP7 project under Grant Agreement 243845 and in part by the Malaysia Ministry of Higher Education. The review of this letter was arranged by Editor R. Quay.

S. R. Kasjoo was with the School of Electrical and Electronic Engineering, University of Manchester, Manchester M13 9PL, U.K. He is now with the School of Microelectronic Engineering, Universiti Malaysia Perlis, Perlis 02600, Malaysia (e-mail: shahrirrizal@unimap.edu.my).

A. M. Song is with the School of Electrical and Electronic Engineering, University of Manchester, Manchester M13 9PL, U.K. (e-mail: a.song@manchester.ac.uk).

Color versions of one or more of the figures in this letter are available online at http://ieeexplore.ieee.org.

Digital Object Identifier 10.1109/LED.2013.2285162
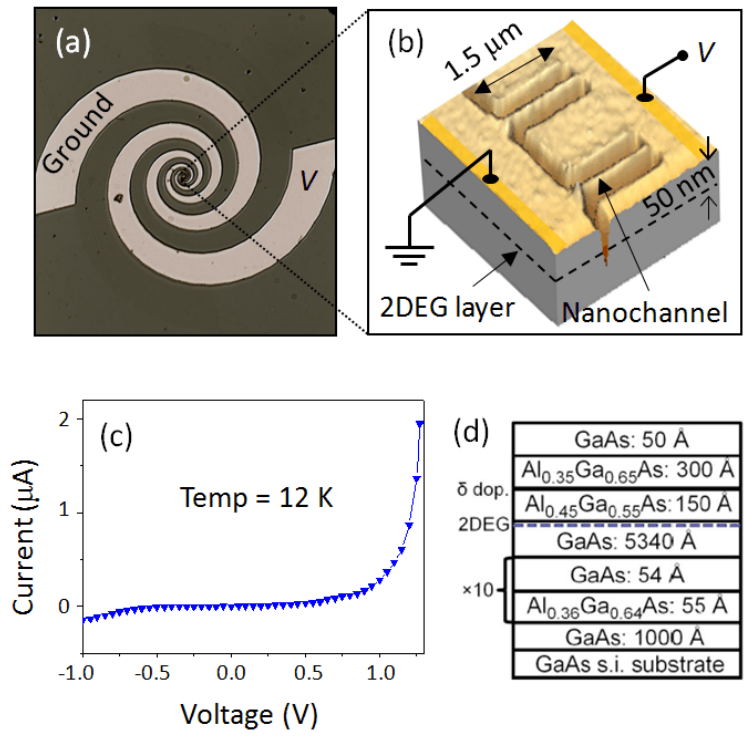

Fig. 1. (a) Optical image of the planar spiral antenna. (b) 3-D rendering of an AFM image of two SSDs (out of four SSDs in total) connected in parallel ( $z$-axis not in scale). The SSD channels were 190-nm wide and 1500-nm long, and the trench width and depth were 150 and $130 \mathrm{~nm}$, respectively. The 2-DEG layer, marked by the dashed line was $50-\mathrm{nm}$ below the surface. (c) DC $I-V$ characteristic of the SSDs at $12 \mathrm{~K}$. (d) Schematic diagram of the material used in the device fabrication.

and characterized the device performance at low temperatures using the $\mathrm{THz}$ radiation generated by a free-electron laser.

The SSD is a unipolar two-terminal device. It consists of two insulating L-shaped trenches opposite to each other, creating a semiconductor nanochannel between them [4]. Fig. 1(b) shows an atomic-force microscope (AFM) image of two SSDs connected in parallel (there were four SSDs in total but only two are shown here for clarity). The applied voltage $V$ to the device can either narrow or widen the effective channel width, depending on its sign [4]. When the device is unbiased $(V=0)$, the effective channel for electrons is pinched off by the surface states on the sidewall of the etched trenches. A negative bias $(V<0)$ further depletes the channel and results in a zero or low current, whereas a positive bias $(V>0)$ counteracts the lateral depletion and widens the effective width of the channel to enable a large current to flow. This results in a diode-like nonlinear currentvoltage $(I-V)$ characteristic as shown in Fig. 1(c), but the SSD is not based on the use of any doped $\mathrm{p}-\mathrm{n}$ junction or Schottky barrier structure and is hence completely different from a conventional diode. This new working principle enables a tunable threshold voltage by simply fabricating a suitable channel width, and hence a zero-threshold may be achieved when the channel is just narrow enough to be fully depleted 
at zero bias [4], [5]. Intrinsically, the SSD possesses a much lower parasitic capacitance when compared with conventional diodes because of its planar architecture in comparison with a multilayered conventional diode of the same size. This enables electrical signal rectification at ultrahigh speed. Because the two electrodes are on the same plane, it is straightforward to integrate coplanar waveguide or planar antenna structures with the SSDs. The truly planar nature of SSD also allows the fabrication of a large number of the devices in a single lithography step without the need for interconnection layers and thus avoiding introducing undesirable parasitic elements [5]-[7].

\section{Device Fabrication And Experimental Setup}

The antenna-coupled-SSD rectifier, i.e., rectenna, was fabricated onto a GaAs/AlGaAs quantum well wafer grown by molecular-beam epitaxy, in which free charge carriers were confined in a quantum well located 50-nm below the surface, forming a 2-D electron gas (2-DEG). The structure of this substrate is shown in Fig. 1(d). The electron mobility and carrier density at a temperature of $77 \mathrm{~K}$ were $72000 \mathrm{~cm}^{2} / \mathrm{Vs}$ and $5.6 \times 10^{11} \mathrm{~cm}^{-2}$, respectively, and at temperature of $300 \mathrm{~K}$ were $7000 \mathrm{~cm}^{2} / \mathrm{Vs}$ and $6.0 \times 10^{11} \mathrm{~cm}^{-2}$, respectively, as determined by Hall measurements.

The first fabrication step was to create mesa structures on the substrate by means of wet chemical etching with a $\mathrm{H}_{3} \mathrm{PO}_{4} / \mathrm{H}_{2} \mathrm{O}_{2} / \mathrm{H}_{2} \mathrm{O}$-based solution. Ohmic contacts on the mesas were formed by thermal evaporation of a $50 \mathrm{~nm}$ of $\mathrm{Au} / \mathrm{Ge} / \mathrm{Ni}$ alloy, followed by $200 \mathrm{~nm}$ of $\mathrm{Au}$, and annealing at $420{ }^{\circ} \mathrm{C}$. A planar spiral microantenna, with a dimension of $\sim 1 \mathrm{~mm}$, was incorporated with the ohmic structures by thermal evaporation of a 200-nm Au layer as shown in Fig. 1(a). Four SSDs connected in parallel were then fabricated in the antenna gap by means of AFM-based lithography, followed by wetchemical etching with a $\mathrm{Br} 2 / \mathrm{HBr} / \mathrm{HNO}_{3} / \mathrm{H}_{2} \mathrm{O}$-based solution. The etched trenches in Fig. 1(b) are $\sim 130$-nm deep, hence through the 2-DEG layer, to ensure electrical insulation. The SSDs channels were 190-nm wide and 1500-nm long, and the trenches width was $150 \mathrm{~nm}$ as determined by the AFM image [Fig. 1(b)]. Further details of the lithography can be found in [8].

Fig. 2 shows the measurement setup used in this letter. A high-power and polarized continuous wave of $\mathrm{THz}$ radiation was generated by a free-electron laser (FELBE at HelmholtzZentrum Dresden-Rossendorf), in which the polarization can be rotated through $90^{\circ}$ by a polarizer. The beam was collimated and guided by a set of flip mirrors arranged on an optical table. By means of an off-axis parabolic mirror, the $\mathrm{THz}$ radiation was focused onto the rectenna that was positioned inside a continuous-flow helium cryostat chamber fitted with a diamond window transparent at $\mathrm{THz}$ frequencies as shown in Fig. 2. Prior to this, the beam was modulated at $120 \mathrm{~Hz}$ by an optical chopper. A dc current source was used to bias the rectenna, and the rectified dc voltage was measured using a lock-in amplifier (Signal Recovery, model 7265 DSP) with the reference frequency input provided by the chopper. The beam intensity was monitored by a pyrometer and can be

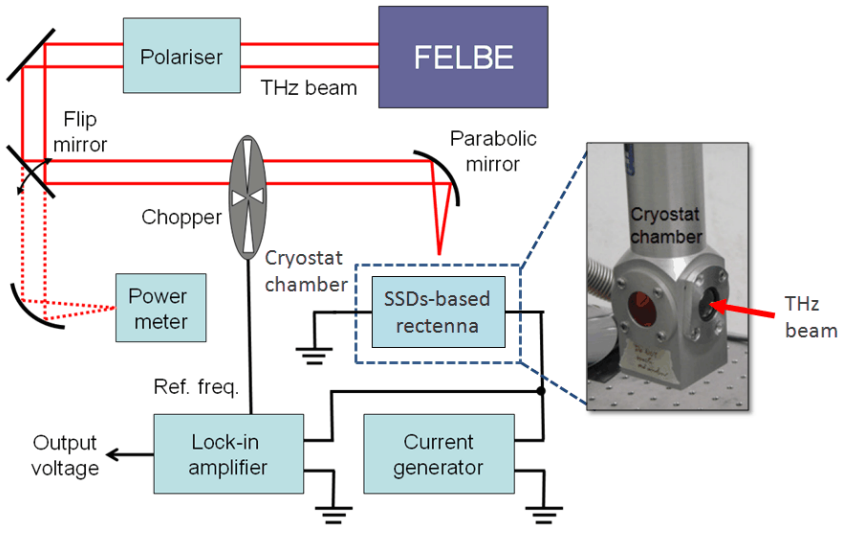

Fig. 2. Diagram showing the measurement setup. The polarized THz radiation produced by FELBE was modulated by an optical chopper, and was focused onto the rectenna, placed inside a cryostat chamber. The device output voltage was read out by a lock-in amplifier with the frequency input provided by the chopper. A tunable dc bias was applied to the device from a current source. A pyrometer was used to estimate the beam power density focused onto the device.

used to estimate the actual power absorbed by the rectenna. The radiation power can also be tuned by adjusting a set of beam attenuators from the control panel that were linked to the FELBE system. This measurement system was almost identical to the setup used in [7] that has verified that the SSDs were only responsive to the radiation collected by the antenna, rather than artifacts such as local heating of the contacts, and so on.

The performance of any receiving antenna used to capture and extract the power from the electromagnetic radiation is usually quantified by the effective aperture, $A_{e}$, defined as the ratio of the power delivered to the load, $P_{\text {deli }}$, to the incident power density, $W_{\text {inci }}$, In this experiment, the power of the $\mathrm{THz}$ radiation, $P_{\mathrm{THz}}$, was measured using a pyrometer. The beam was then focused onto the rectenna by means of parabolic mirrors. By considering, the focused beam incident on the device has a circular area with a radius $r$, the value of $W_{\text {inci }}$ can be estimated as $P_{\mathrm{THz}} /\left(\pi r^{2}\right)$. For a receiving antenna, the value of $A_{e}$ is also related to its gain, $G$, by the following equation, $A_{e}=G \lambda^{2} /(4 \pi)$, where $\lambda$ is the wavelength of the electromagnetic wave. Therefore, $P_{\text {deli }}$ can be estimated as

$$
P_{\text {deli }}=G\left(\frac{\lambda}{2 \pi r}\right)^{2} P_{\mathrm{THz}}
$$

\section{RESULTS AND DISCUSSION}

Fig. 3(a) shows the rectified dc output voltage, $V_{\text {out }}$ as a function of the bias current, $I_{\text {bias}}$, at $1.5 \mathrm{THz}(\lambda=200 \mu \mathrm{m})$. The measurement was carried out at a range of THz-beam power, $P_{\mathrm{THz}}$ and at a temperature of $12 \mathrm{~K}$ that was the lowest temperature at which the cryostat could maintain a good stability. Through considering the beam diameter and the antenna effective aperture, the power delivered to the spiral antenna (i.e., $P_{\text {deli }}$ ) can be estimated from (1). Here, $r=1 \mathrm{~mm}$ as determined by mechanically shifting the SSDs in and out the focus point of the parabolic mirror, and $G=2$ was used here for the spiral microantenna based on the simulations performed in [9]. 

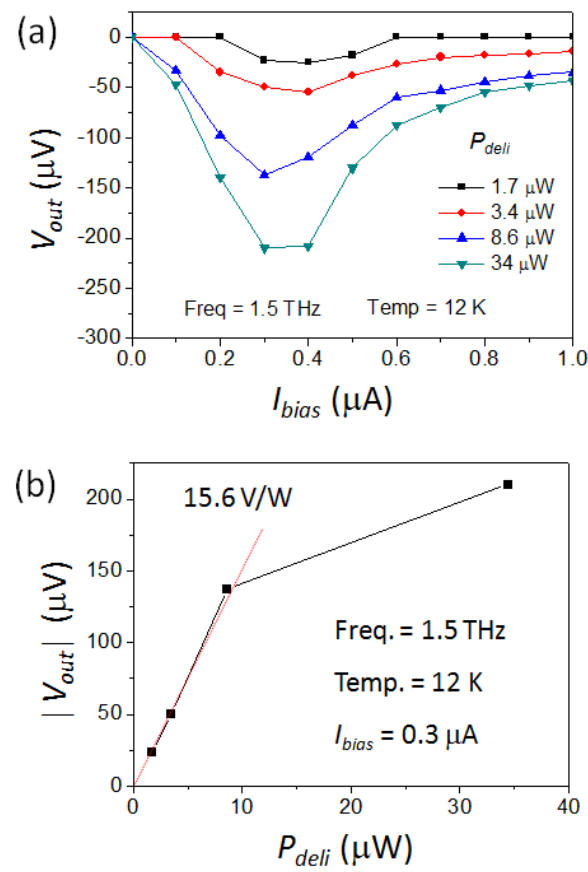

Fig. 3. (a) $V_{\text {out }}$ as a function of $I_{\text {bias }}$, measured at $12 \mathrm{~K}$ at $1.5 \mathrm{THz}$ at different values of $P_{\text {deli }}$. The highest magnitude of $V_{\text {out }}$ was obtained at $I_{\text {bias }}$ around $0.3-0.4 \mu \mathrm{A}$. (b) $V_{\text {out }}$ as a function of $P_{\text {deli }}$, measured at $12 \mathrm{~K}$ at $1.5 \mathrm{THz}$ with $I_{\text {bias }}=0.3 \mu \mathrm{A}$. A linear response (square-law detection) was observed when $P_{\text {deli }}<10 \mathrm{~W}$. In this region, the device extrinsic voltage responsivity was $15.6 \mathrm{~V} / \mathrm{W}$, estimated from the fitting line (dotted line).

The amount of $V_{\text {out }}$ rectified by the SSDs is dependent on the nonlinearity of the $I-V$ characteristic (bowing coefficient) and differential resistance of the SSDs [6]. The bowing coefficient increases with increasing $I_{\text {bias }}$ up to certain bias level and then slowly decreases because of the parasitic resistance in series with the SSDs as shown in Fig. 1(c). The differential resistance of the SSDs decreases as $I_{\text {bias }}$ increases. As a result, the combination of both parameters with respect to $I_{\text {bias }}$ yielded the THz responsivity curves in Fig. 3(a). As can be seen, similar traces were observed at different $P_{\text {deli }}$, and the highest magnitude of $V_{\text {out }}$ for each measurement was achieved at $I_{\text {bias }}$ of $0.3-0.4 \mu \mathrm{A}$. This was the region where $I-V$ characteristic of the SSDs exhibited the strongest nonlinearity.

Fig. 3(b) shows the measurement of $V_{\text {out }}$ as a function of $P_{\text {deli }}$ at $12 \mathrm{~K}$ with $I_{\text {bias }}=0.3 \mu \mathrm{A}$. As expected, the rectenna displayed a linear response (square-law detection) at power levels lower than $10 \mu \mathrm{W}$. In this region, the voltage responsivity, $\beta_{V}$, of the device was $\sim 15.6 \mathrm{~V} / \mathrm{W}$.

Such responsivity is extrinsic, which is largely limited by the impedance mismatch between the SSDs and the antenna. The simulations (not shown here) show that the antenna with a GaAs substrate has a impedance of $\sim 100 \Omega$ at frequencies $<1 \mathrm{THz}$ and $\sim 50 \Omega$ at $1.5 \mathrm{THz}$. In contrast, the impedance of the SSDs at $I_{\text {bias }}=0.3 \mu \mathrm{A}$ at $12 \mathrm{~K}$ was $\sim 600 \mathrm{k} \Omega$. This means that only $\sim 0.033 \%$ of $P_{\text {deli }}$ was effectively applied to the SSDs with nearly all the incident power being reflected.
Taking into account this factor, the intrinsic voltage responsivity of the device can achieve up to $45 \mathrm{kV} / \mathrm{W}$. Commercial low-temperature bolometers typically have a responsivity in the order of a few kilovolt/watt to $10 \mathrm{kV} / \mathrm{W}$ [10], [11]. By optimization of the SSD technologies, such as fabricating more SSDs in parallel to reduce the overall impedance [5], [12], filling the trenches with high- $k$ materials [13], and designing higher-impedance waveguide/antenna structures to couple to the SSDs, the performance of the SSDs is expected to be greatly improved. It is worth noticing that the highest frequency that we could utilize in this experiment was $1.5 \mathrm{THz}$, which might not be the upper operational limit of the SSDs, as previously predicted by Monte Carlo simulations [14]. The advantage of the truly planar nature to allow straightforward integration with coplanar waveguide or planar antenna structures with the SSDs offers a great potential for low-cost $\mathrm{THz}$ detection applications.

\section{ACKNOWLEDGMENT}

The authors would like to thank M. McGowan, Dr. C. Balocco, Dr. X. F. Lu, Dr. S. Winnerl, and HZDR for technical assistance and the use of FELBE facilities.

\section{REFERENCES}

[1] A. Redo-Sanchez and X. C. Zhang, "Terahertz science and technology trends," IEEE J. Sel. Topics Quantum Electron., vol. 14, no. 2, pp. 260-269, Mar./Apr. 2008.

[2] T. Nagatsuma, "Terahertz technologies: Present and future," IEICE Electron. Exp., vol. 8, no. 14, pp. 1127-1142, Jul. 2011.

[3] P. Knobloch, C. Schildknecht, T. Kleine-Ostmann, et al., "Medical THz imaging: An investigation of histo-pathological samples," Phys. Med. Biol., vol. 47, no. 21, pp. 3875-3884, Oct. 2002.

[4] A. M. Song, M. Missous, P. Omling, et al., "Unidirectional electron flow in a nanometer-scale semiconductor channel: A self-switching device," Appl. Phys. Lett., vol. 83, no. 9, pp. 1881-1883, Jul. 2003.

[5] C. Balocco, A. M. Song, M. Åberg, et al., "Microwave detection at $110 \mathrm{GHz}$ by nanowires with broken symmetry," Nano Lett., vol. 5, no. 7, pp. 1423-1427, Jun. 2005.

[6] C. Balocco, M. Halsall, N. Q. Vinh, et al., "THz operation of asymmetric-nanochannel devices," J. Phys., Condens. Matter., vol. 20, no. 38, pp. 384203-384207, Aug. 2008

[7] C. Balocco, S. R. Kasjoo, X. F. Lu, et al., "Room-temperature operation of a unipolar nanodiode at terahertz frequencies," Appl. Phys. Lett., vol. 98, no. 22, pp. 223501-1-223501-3, May 2011.

[8] X. F. Lu, C. Balocco, F. H. Yang, et al., "Highly reproducible nanolithography by dynamic plough of an atomic-force microscope tip and thermally-annealing treatment," IEEE Trans. Nanotechnol., vol. 10, no. 1, pp. 53-58, Jan. 2011.

[9] S. H. Choudhry, "Antenna design for terahertz nanodevices," M.S. thesis, School of Electr. Electron. Eng., Univ. Manchester, Manchester, U.K., 2009.

[10] Bolometer Products [Online]. Available: http://www.terahertz.co.uk/

[11] F. Sizov and A. Rogalski, "THz detectors," Progr. Quantum Electron., vol. 34, no. 5, pp. 278-347, Sep. 2010.

[12] P. Sangaré, G. Ducournau, B. Grimbert, et al., "Experimental demonstration of direct terahertz detection at room temperature in AlGaN/GaN asymmetric nanochannels," J. Appl. Phys., vol. 113, no. 3, pp. 034305-1-034305-6, Jan. 2013.

[13] M. Y. Irshaid, C. Balocco, Y. Luo, et al., "Zinc-oxide-based planar nanodiodes operating at $50 \mathrm{MHz}$,' Appl. Phys. Lett., vol. 99, no. 9, pp. 092101-1-092101-3, Aug. 2011.

[14] J. Mateos, B. G. Vasallo, D. Pardo, et al., "Operation and high-frequency performance of nanoscale unipolar rectifying diodes," Appl. Phys. Lett., vol. 86, no. 21, pp. 212103-212105, May 2005. 